\title{
Nota científica \\ CARACTERÍSTICAS DEL SUSTRATO Y CALIDAD DE PLANTINES DE LECHUGA (Lactuca sativa L.) SEGÚN DOSIS DE LOMBRICOMPUESTO
}

\section{Scientific note \\ SUBSTRATE CHARACTERISTICS AND LETTUCE (Lactuca sativa \\ L.) SEEDLING QUALITY DEPENDING ON THE VERMICOMPOST DOSE}

\author{
Leticia Tombion ${ }^{1}$, Analia V. Puerta ${ }^{1}$, Lorena A. Barbaro ${ }^{2}$, Mónica A. Karlanian ${ }^{2}$, Miguel A. \\ Sangiacomo ${ }^{1}$, y Mariana Garbi ${ }^{*}$ \\ ${ }^{1}$ Universidad Nacional de Luján, Depto. de Tecnología, Rutas 5 y 7, Luján, Buenos Aires, Argentina. \\ 2 Instituto de Floricultura del INTA, De los Reseros y Las Cabañas (CP1686), Castelar, Buenos Aires, \\ * Autor para correspondencia E-mail: mgarbi@mail.unlu.edu.ar
} Argentina.

\section{RESUMEN}

El uso de sustrato con lombricompuesto favorece el crecimiento en cultivos hortícolas, siendo importante conocer sus propiedades para dosificar la cantidad adecuada. Este trabajo tuvo como objetivo analizar las modificaciones producidas en un sustrato según la dosis de lombricompuesto adicionado y evaluar el efecto de las mezclas sobre la calidad del plantín de lechuga. El ensayo se realizó en un invernadero en Luján, Argentina $\left(34^{\circ} 36^{\prime} \mathrm{S}, 5^{\circ} 04^{\prime} \mathrm{W}\right)$. Se sembró lechuga mantecosa cv. Tizona, utilizando sustratos compuestos por distintas combinaciones porcentuales de lombricompuesto y una mezcla comercial formulada con turba y perlita: 1) $0 \%$ lombricompuesto, 2) $20 \%$ lombricompuesto, 3) $40 \%$ lombricompuesto. Las mezclas se analizaron físicoquímicamente. En las plantas se midió número de hojas, peso fresco y seco de parte aérea, raíz y total. El diseño fue en bloques completos aleatorizados con 4 repeticiones. Se realizó análisis de la varianza, evaluando diferencias entre medias por la prueba de Tukey y análisis de regresión para estudiar el tipo de respuesta en los parámetros físicoquímicos de los sustratos por la incorporación de lombricompuesto en el rango de 0 a $40 \%$. La adición de lombricompuesto en los sustratos aumentó linealmente el $\mathrm{pH}$, la conductividad eléctrica, el nivel de nutrientes, la densidad aparente y la porosidad total, y disminuyó linealmente la materia orgánica y cuadráticamente el porcentaje de poros ocupados por agua. Las plantas respondieron al agregado de lombricompuesto adquiriendo mayor tamaño con $20 \%$.

Palabras clave: almácigo, vermicompuesto, Eisenia foetida.

\section{ABSTRACT}

Substrates with vermicompost added promote growth in horticultural crops. Therefore, it is important to know its properties in order to apply proper amounts of it. This work aims to analyze substrate changes according to vermicompost doses and evaluate the effect of the mixtures on lettuce seedling quality. The essay was carried out under greenhouse conditions in Luján, Argentina $\left(34^{\circ} 36^{\prime} \mathrm{S}, 5^{\circ} 04^{\prime} \mathrm{W}\right)$. Butterhead lettuce $\mathrm{cv}$. Tizona was sown using substrates with different amounts of vermicompost added and a commercial mix of peat and perlite: 1) $0 \%$ vermicompost, 2) $20 \%$ vermicompost, 3) $40 \%$ vermicompost. The substrates were analyzed physically and chemically. The 
number of leaves and both fresh and dry weights of leaves, roots and plant were determined. The treatments were established in a complete randomized block design with four replications. Data were evaluated by analysis of variance, while the difference between means was studied by Tukey`s Test. Regression analysis was used to study the response of substrate physical and chemical parameters to the addition of vermicompost, ranging from 0 to $40 \%$. The addition of vermicompost resulted in a linear increased in $\mathrm{pH}$, electrical conductivity, nutrient level, bulk density and total porosity of the substrate, while organic matter decreased linearly and quadratically the percentage of pores occupied by water. The plants responded to the addition of vermicompost, with a $20 \%$ increase in size.

Key words: nursery, earthworm, Eisenia foetida.

\section{INTRODUCCIÓN}

El lombricompuesto es un material estabilizado, libre de olores y con características deseables como componente de sustratos de cultivo en distintos del suelo, que se obtiene por la descomposición de residuos orgánicos a partir de la acción conjunta de las lombrices de tierra (Eisenia foetida) y distintos microorganismos (Paul y Metzger, 2005; Melgar-Ramírez y Pascual-Alex, 2010; Basheer y Agrawal, 2013). Entre sus características fundamentales se encuentran su buena estructura, drenaje, aireación, capacidad de retención de humedad, abundante cantidad de nutrientes en formas fácilmente asimilables para las plantas; además de la presencia de partículas cuya área superficial favorece la retención de los nutrientes, y provee micrositios aptos para la actividad de especies microbianas con comprobada aptitud para la generación de sustancias con acción promotora del crecimiento, del tipo de las auxinas, giberelinas y ácidos húmicos (Aaloket al., 2008; Argüello et al., 2013).

La incorporación de lombricompuesto a las mezclas de siembra favorece el crecimiento y producción en diversos cultivos hortícolas, como ha sido observado en tomate (Solanum lycopersicum), lechuga (Lactuca sativa L.), ajo (Allium sativum L.) y frutilla (Fragaria vesca L.), entre otras especies (Domínguez et al., 2010). En tomate, Tringovska y Dintcheva (2012) evaluaron la respuesta de la planta frente a la utilización de lombricompuesto procedente de residuos de distintos orígenes, concluyendo que el agregado de este material a la mezcla, indistintamente de su origen, favoreció el crecimiento, lo que podría atribuirse al aporte de macronutrientes, pero fundamentalmente a la modificación de las propiedades físicas y biológicas de las mezclas resultantes. En lechuga, la incorporación de lombricompuesto al sustrato produjo mayor crecimiento de los plantines e incremento de la biomasa aérea (López et al., 1999; Argüello et al., 2013).

El efecto más positivo sobre el crecimiento de las plantas se obtuvo con la incorporación de 10 a $20 \%$ de lombricompuesto a la mezcla. En tomate se ha observado un incremento en el peso seco del plantín al incorporar un $20 \%$ de lombricompuesto, mientras que en berenjena (Solanum melongena L.) el mayor crecimiento se observó con el agregado de 10\% (Paul y Metzger, 2005). De Grazia et al. (2006) consideran que el lombricompuesto causa resultados satisfactorios sobre el crecimiento de las hortalizas, aunque sostienen que si este abono orgánico conforma sustratos nutricionalmente muy enriquecidos, puede ser perjudicial debido a su alto contenido de sales solubles. Este efecto fue observado por Tatlari et al. (2013) quienes reportaron una respuesta negativa de la planta en Dracaena marginata, al incorporar al sustrato cantidades crecientes de lombricompuesto, lo que producía la elevación fuera de los rangos aceptables para la especie del nivel de conductividad eléctrica del medio de crecimiento.

Bárbaro et al. (2011a) destacan que entre los principales problemas de los productores de plantas en contenedor se encuentra el desconocimiento de las características de los materiales utilizados en las mezclas para siembras; mientras que Valenzuela et al. (1998) y Castillo Taco (2010) señalan que es de suma importancia conocer las propiedades físicas y químicas del humus de lombriz antes de su utilización, para descartar aspectos perjudiciales para el cultivo, y dosificar una cantidad de abono que no dañe los procesos fisiológicos de la planta.

El objetivo de este trabajo fue analizar las modificaciones producidas en un sustrato de siembra formulado en base a turba y perlita según la dosis de lombricompuesto adicionado, y evaluar el efecto de las mezclas resultantes sobre la calidad de la planta de lechuga obtenida al momento de trasplante.

\section{MATERIALES Y MÉTODOS}

El ensayo se realizó en un invernadero parabólico ubicado en el Campo Experimental de la Universidad Nacional de Luján, Luján, Buenos Aires ( $\left.34^{\circ} 36^{\prime} \mathrm{S}, 5^{\circ} 04^{\prime} \mathrm{O}\right)$. Se utilizó lechuga mantecosa cv. Tizona (Florenza ${ }^{\circledR}$ ) sembrada en bandejas de germinación de polipropileno negro 
de 200 celdas con capacidad de $11 \mathrm{~cm}^{3}$. La siembra se realizó el 10/08/2013, utilizando tres sustratos compuestos por distintas combinaciones porcentuales de un lombricompuesto (Lombriscomp, Bertinat ${ }^{\circledR}$, Bella Vista, Buenos Aires, Argentina), elaborado comercialmente y una mezcla comercial para siembra y repique (Bertintat ${ }^{\circledR}$, Bella Vista, Buenos Aires), formulada con turba y perlitacomo componentes principales. Las características del lombricompuesto se presentan en la Tabla 1, y las de la mezcla comercial para siembra y repique en la Tabla 2.

Se realizaron los siguientes tratamientos: 1) $0 \%$ de lombricompuesto, 2) $20 \%$ de lombricompuesto y 3) $40 \%$ de lombricompuesto. El lombricompuesto y las formulaciones utilizadas en los tres tratamientos se analizaron por triplicado para su caracterización física y química, determinándose materia orgánica con el método mufla (Ansorena, 1994), densidad aparente por el método de Hofman (Fermino, 2003), espacio poroso total, capacidad de retención de agua y poros con aire (De Boodt et al., 1974), pH y conductividad eléctrica en 1+5 vol/vol (Bárbaro et al., 2011b), la concentración de calcio, magnesio, potasio y sodio se analizó por filtrado de solución $1+5$ con un espectrómetro de absorción atómica (Varian modelo 220 A, Palo Alto, California, Estados Unidos de América) y los nitrato con electrodo ión selectivo (Orion modelo 920 A, Waltham, Massachusetts, Estados Unidos de América).

Se utilizó un diseño en bloques completos aleatorizados con 4 repeticiones. Se tomaron al azar 10 plantas por cada tratamiento y repetición, y se realizaron las siguientes determinaciones: número de hojas, contando las hojas expandidas mayores a $1 \mathrm{~cm}$ de longitud; peso fresco de la parte aérea, de la raíz y de la planta entera, utilizando una balanza analítica; peso seco de la parte aérea, de la raíz y de la planta entera, por secado en estufa a $70-80^{\circ} \mathrm{C}$ y pesado en balanza analítica.

Los datos se sometieron a análisis de la varian$\mathrm{za}$, evaluando las diferencias entre medias por la prueba de Tukey. Se utilizó el análisis de regresión para evaluar el tipo de respuesta de los parámetros físicoquímicos de los sustratos y en las plantas por la incorporación de lombricompuesto a la formulación de siembra, en el rango de $0 \%$ a $40 \%$. Los análisis estadísticos se realizaron con el programa Infostat (Di Rienzo et al., 2013).

\section{RESULTADOS Y DISCUSIÓN}

La caracterización física y química de los tres sustratos evaluados en este trabajo se presenta en la Tabla 2 y el tipo de respuesta de cada variable a la incorporación de lombricompuesto a la mezcla, en el rango de $0 \%$ a $40 \%$, en la Tabla 3.

La incorporación de lombricompuesto en el sustrato incrementó en forma estadísticamente significativa el $\mathrm{pH}$, la conductividad eléctrica y el contenido en todos los elementos nutritivos analizados, observándose diferencias entre todos los tratamientos, con respuestas de tipo linear en este incremento. Tringovska y Dintcheva (2012) también encontraron aumentos en estas variables, al estudiar el efecto de la incorporación de $10 \%$ de lombricompuestos de distintos orígenes y procesos de obtención a una mezcla compuesta por turba y perlita; mientras que la incorporación de proporciones crecientes de un material compostado por digestión anaeróbica también produjo incrementos en los valores de $\mathrm{pH}$ y conductividad eléctrica en los sustratos formulados con una

Tabla 1. Caracterización física y química del lombricompuesto adicionado a la mezcla comercial compuesta por turba y perlita.

Table 1. Physical and chemical characteristics of vermicompost added to a commercial mix of peat and perlite.

\begin{tabular}{|c|c|}
\hline $\mathrm{pH}$ & 7,10 \\
\hline C.E., $\mathrm{dS} \mathrm{m}^{-1}$ & 2,89 \\
\hline Nitratos, $\mathrm{mg} \mathrm{L}^{-1}$ de muestra & 2485 \\
\hline Calcio, $\mathrm{mg} \mathrm{L}^{-1}$ de muestra & 13657 \\
\hline Magnesio, $\mathrm{mg} \mathrm{L}^{-1}$ de muestra & 1093 \\
\hline Potasio, $\mathrm{mg} \mathrm{L}^{-1}$ de muestra & 6565 \\
\hline Sodio, $\mathrm{mg} \mathrm{L}^{-1}$ de muestra & 1468 \\
\hline Densidad aparente, $\mathrm{kg} \mathrm{m}^{-3}$ & 308 \\
\hline Materia orgánica, $\%$ en masa & 40 \\
\hline Porosidad de aire, $\%$ en volumen & 25 \\
\hline Porosidad de agua, $\%$ en volumen & 59 \\
\hline Porosidad total, $\%$ en volumen & 84 \\
\hline
\end{tabular}


Tabla 2. Caracterización física y química de los sustratos según la proporción de lombricompuesto adicionado a la mezcla comercial compuesta por turba y perlita.

Table 2. Physical and chemical characteristics of substrates depending on the amount of vermicompost added to a commercial mix of peat and perlite.

\begin{tabular}{|c|c|c|c|c|c|}
\hline \multicolumn{6}{|c|}{ Proporción de lombricompuesto } \\
\hline & 0 & 20 & 40 & C.V. & $\mathbf{R}^{2}$ \\
\hline \multicolumn{6}{|c|}{--------------- \% - --------------- } \\
\hline $\mathrm{pH}$ & $4,47 \mathrm{a}$ & $5,98 \mathrm{~b}$ & $6,47 \mathrm{c}$ & 0,81 & 1,00 \\
\hline C.E., dS m ${ }^{-1}$ & $0,22 \mathrm{a}$ & $1,36 \mathrm{~b}$ & $2,04 \mathrm{c}$ & 2,80 & 1,00 \\
\hline Nitratos, $\mathrm{mg} \mathrm{L}^{-1}$ de muestra & $473 \mathrm{a}$ & $1122 \mathrm{~b}$ & 1492 c & 2,13 & 1,00 \\
\hline Calcio, $\mathrm{mg} \mathrm{L}^{-1}$ de muestra & $102 \mathrm{a}$ & $2587 \mathrm{~b}$ & $6641 \mathrm{c}$ & 18,50 & 0,97 \\
\hline Magnesio, $\mathrm{mg} \mathrm{L}^{-1}$ de muestra & $47 \mathrm{a}$ & $432 \mathrm{~b}$ & $774 \mathrm{c}$ & 2,89 & 1,00 \\
\hline Potasio, $\mathrm{mg} \mathrm{L}^{-1}$ de muestra & $84 \mathrm{a}$ & $1722 \mathrm{~b}$ & $3676 \mathrm{c}$ & 3,37 & 1,00 \\
\hline Sodio, $\mathrm{mg} \mathrm{L}^{-1}$ de muestra & $45 \mathrm{a}$ & $730 \mathrm{~b}$ & $951 \mathrm{c}$ & 4,08 & 1,00 \\
\hline Densidad aparente, $\mathrm{kg} \mathrm{m}^{-3}$ & $0,13 \mathrm{a}$ & $0,17 \mathrm{~b}$ & $0,21 \mathrm{c}$ & 2,77 & 0,98 \\
\hline Materia orgánica, \% en masa & 58 a & $52 \mathrm{~b}$ & $50 \mathrm{c}$ & 1.08 & 0,98 \\
\hline Porosidad de aire, \% en volumen & 12 & $11 \mathrm{a}$ & $18 \mathrm{~b}$ & 13,64 & 0,75 \\
\hline Porosidad de agua, \% en volumen & 79 & 78 & $71 \mathrm{~h}$ & 263 & 0,86 \\
\hline Porosidad total \% en volumen & 9? & 91 a & $89 \mathrm{~b}$ & $\begin{array}{l}2,00 \\
037\end{array}$ & 0,95 \\
\hline & $92 \mathrm{a}$ & $91 \mathrm{a}$ & & & \\
\hline
\end{tabular}

Letras diferentes en la fila indican diferencias estadísticamente significativas según Test de Tukey $(\mathrm{p}<0,05)$

Tabla 3. Tendencia de la respuesta de los parámetros físicos y químicos de los sustratos a la incorporación de lombricompuesto a la mezcla en el rango de $0 \%$ a $40 \%$.

Table 3. Response trend of the substrate physicochemical parameters to the addition of vermicompost in the range from $0 \%$ to $40 \%$.

\begin{tabular}{lrrr}
\hline & Tendencia & \multicolumn{1}{c}{$\mathbf{P}$} & $\mathbf{R}^{\mathbf{2}}$ \\
\hline $\mathrm{pH}$ & Linear & $<0,0001$ & 0,92 \\
$\mathrm{C}$. E. & Linear & $<0,0001$ & 0,98 \\
Nitratos & Linear & $<0,0001$ & 0,97 \\
Calcio & Linear & $<0,0001$ & 0,95 \\
Magnesio & Linear & $<0,0001$ & 1,00 \\
Potasio & Linear & $<0,0001$ & 0,92 \\
Sodio & Linear & $<0,0001$ & 1,00 \\
Densidad aparente & Linear & $<0,0001$ & 0,98 \\
Materia orgánica & Linear & 0,0001 & 0,91 \\
Porosidad de aire & Cuadrática & 0,0045 & 0,71 \\
Porosidad de agua & Cuadrática & 0,0005 & 0,84 \\
Porosidad total & Linear & $<0,0001$ & 0,95 \\
\hline
\end{tabular}

base de turba (Restrepo et al., 2013). El porcentaje de humedad y contenido de materia orgánica decrecieron linealmente y en forma estadísticamente significativa al aumentar la proporción de lombricompuesto, coincidiendo con lo observado por Restrepo et al. (2013).

Las características físicas del sustrato también varían por la incorporación de lombricompuesto, como reportaron Melgar-Ramirez y Pascual-Alex
(2010) quienes, al comparar mezclas con proporciones crecientes de lombricompuesto observaron un aumento de la densidad aparente y una disminución de la porosidad total, que coincide con los resultados obtenidos en esta investigación. Los mismos autores observaron que en los sustratos con mayor cantidad de lombricompuesto en su composición se aumentaba la porosidad de aireación, habiéndose observado una respuesta 
similar en las condiciones de este ensayo. El sustrato con $40 \%$ de lombricompuesto presentó una proporción de poros ocupados por agua significativamente menor y un aumento en la cantidad de poros de aireación, modificación atribuible al mayor porcentaje de porosidad de aire presente en el lombricompuesto, en relación a la mezcla comercial para siembra y repique, lo que mejoró las características de la mezcla final. La variación mencionada en la porosidad total, producto de la incorporación de $40 \%$ lombricompuesto, no afecta la calidad del sustrato en este sentido, dado que los valores registrados se encuentran por encima del valor óptimo de referencia, que debe ser superior al 85\% (Restrepo et al., 2013).

El número de hojas, el peso fresco de la planta y sus partes constituyentes y el peso seco de la raíz fueron significativamente ( $p \leq 0,0001)$ más elevados en plantas cultivadas con $20 \%$ de lombricompuesto, diferenciándose de las obtenidas en sustratos con $40 \%$; mientras que en el peso seco de la parte aérea y de la planta esta diferencias se observó también respecto al sustrato sin lombricompuesto (Tabla 4). Un efecto negativo de la incorporación de lombricompuesto sobre el peso seco de raíces fue también observado por Paul y Metzger (2005) en plantines de tomate (Solanum lycopersicum), pimiento (Capsicum annum L.) y berenjena (Solanum melongena L.) cultivados con $10 \%$ de lombricompuesto, al compararlos con plantas obtenidas en un sustrato sin lombricompuesto. Argüello et al. (2013) determinaron en lechuga (Lactuca sativa L.) que la acción del lombricompuesto sobre el crecimiento se da por su efecto sobre el meristema fundamental de la hoja, observándose mayor espesor y número de capas del clorénquima y modificaciones anatómicas que incrementan la biomasa y la distribución de fotoasimilados, lo que explicaría el mayor incremento en peso de la parte aérea de la planta por la incorporación de $20 \%$ lombricompuesto a la mezcla, en detrimento de la raíz.

La riqueza en nutrientes que aporta la incorporación del lombricompuesto al sustrato es uno de los mecanismos que podrían explicar el efecto positivo de este elemento sobre el crecimiento de plantines (Paul y Metzger, 2005). En este ensayo, todos los elementos nutritivos incrementaron linealmente su cantidad con incorporaciones crecientes de lombricompuesto, lo que también repercutió en el nivel de conductividad eléctrica,

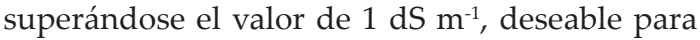
las mezclas utilizadas como sustrato (Bárbaro et al., 2014). La mezcla con $20 \%$ de lombricompuesto presentó un valor cercano al tolerado y su efecto sobre el crecimiento de la planta fue positivo, lo que pudo deberse a que el nivel de conductividad eléctrica alcanzado $\left(1,36 \mathrm{dS} \mathrm{m}^{-1}\right)$ se encuentra en el rango de los valores umbrales para la especie, que oscila entre 1,0 y $1,4 \mathrm{dS} \mathrm{m}^{-1}$, pudiendo existir diferencias varietales en esta tolerancia (Carranza et al., 2009). En el caso del pH, el rango adecuado para sustratos se ubica en valores de 5,2 a 6,3 (Noguera et al., 2003), niveles que se alcanzan con la incorporación de 20 a 40\% de lombricompuesto a la mezcla, pero que se superarían con proporciones mayores, dada la respuesta linear observada en el incremento de esta variable.

\section{CONCLUSIONES}

La incorporación de $20 \%$ de lombricompuesto a la mezcla de siembra mejoró las características del sustrato y las condiciones de crecimiento para la planta. Se evidencia la importancia de controlar adecuadamente la proporción de lombricompuesto a utilizar, atendiendo los requerimientos de la especie; recomendándose considerar al

Tabla 4. Características de plantines de lechuga mantecosa cv. Tizona según la proporción de lombricompuesto adicionado a la mezcla comercial compuesta por turba y perlita.

Table 4. Characteristics of butterhead lettuce seedlings cv. Tizona depending on the amount of vermicompost added to a commercial mix of peat and perlite.

\begin{tabular}{|c|c|c|c|c|c|c|c|}
\hline \multirow{2}{*}{$\begin{array}{l}\text { Lombri- } \\
\text { compuesto }\end{array}$} & \multirow[b]{2}{*}{ № hojas } & \multicolumn{3}{|c|}{ Peso fresco } & \multicolumn{3}{|c|}{ Peso seco } \\
\hline & & Hoja & Raíz & Planta & Hoja & Raíz & Planta \\
\hline & & \multicolumn{6}{|c|}{ - } \\
\hline $0 \%$ & $5 a b$ & $0,147 \mathrm{ab}$ & $0,076 \mathrm{ab}$ & $0,223 \mathrm{ab}$ & $0,018 \mathrm{a}$ & $0,015 \mathrm{ab}$ & $0,033 \mathrm{a}$ \\
\hline $20 \%$ & $6 \mathrm{~b}$ & 0,247 a & 0,090 a & 0,337 a & $0,032 \mathrm{~b}$ & $0,021 \mathrm{a}$ & $0,053 \mathrm{~b}$ \\
\hline $40 \%$ & $4 \mathrm{a}$ & $0,120 \mathrm{~b}$ & $0,031 \mathrm{~b}$ & $0,151 \mathrm{~b}$ & $0,012 \mathrm{a}$ & $0,009 \mathrm{~b}$ & $0,021 \mathrm{a}$ \\
\hline C.V. & 7,11 & 28,85 & 35,59 & 22,88 & 19,44 & 19,42 & 18,92 \\
\hline $\mathrm{R}^{2}$ & 0,84 & 0,76 & 0,75 & 0,85 & 0,90 & 0,85 & 0,79 \\
\hline
\end{tabular}

Letras diferentes en la columna indican diferencias estadísticamente significativas $(p<0,05)$ 
menos valores de $\mathrm{pH}$ y conductividad eléctrica, parámetros fácilmente obtenibles, para evitar condiciones que pueden resultar altamente perjudiciales para la obtención de plantines de calidad.

\section{LITERATURA CITADA}

Aalok, A., A.K. Tripathi, y P. Soni. 2008. Vermicomposting: A better option for organic solid waste management. Journal of Human Ecology 24(1):59-64.

Ansorena, M.J. 1994. Sustratos propiedades y caracterización. Mundi-Prensa, Madrid, España.

Argüello, J.A., L. Seisdedos, M.C. Díaz Goldfarb, E.A. Fabio, S.B. Núñez, y A. Ledesma. 2013. Modificaciones anatomofisiológicas inducidas por residuos sólidos agrícolas (vermicompuesto) en plantines de lechuga (Lactuca sativa L.). ФYTON Revista Internacional de Botánica Experimental 82:289-295.

Bárbaro, L.A., M.A. Karlanian, O. Valenzuela, C. Gallardo, J. González, C.K. Del Pardo, et al. 2011a. Caracterización y evaluación de sustratos de siembra utilizados para el cultivo de especies ornamentales. Disponible en http://inta.gob.ar/documentos/caracterizacion-y-evaluacion-de-sustratos-de-siembra-utilizados-para-el-cultivo-de-especies-ornamentales-1/ (Consulta 9 de mayo de 2014).

Bárbaro, L.A., M.A. Karlanian, S. Imhoff, y D. Morisigue. 2011b. Caracterización de la turba subtropical del departamento Islas del Ibicuy (Entre Ríos, Argentina). Agriscientia 28(2):137-145.

Barbaro, L.A.; A. Mazzoni, M.A. Karlanian, M.N. Fernandez y Morisigue, D.E. 2014 Cenizas del volcán Puyehue como sustrato para plantas. Horticultura Argentina 33(81):44-53.

Basheer, M., y O.P. Agrawal. 2013. Effect of vermicompost on the growth and productivity of tomato plant (Solanum lycopersicum) under field conditions. International Journal of Recent Scientific Research (4)3:247-249.

Carranza, C., O. Lanchero, D. Miranda, y B. Chaves. 2009. Análisis del crecimiento de lechuga (Lactuca sativa L.) 'Batavia' cultivada en un suelo salino de la Sabana de Bogotá. Agronomía Colombiana 27(1):41-48.

Castillo Taco, J.C. 2010. Análisis de lombricompuestos a partir de diferentes sustratos. Tesis Especialización en Cultivos Perennes Industriales. Escuela de Postgrados, Facultad de Agronomía, Universidad Popular del Cesar, Universidad Nacional de Colombia, Valledupar, Colombia.
De Boodt, M., O. Verdonck, y I. Cappaert. 1974. Method for measuring the water release curve of organic substrates. Acta Horticulturae 37:2054-2062.

De Grazia, J., P.A. Tittonell, y A. Chiesa. 2006. Efecto de sustratos con compost y fertilización nitrogenada sobre la fotosíntesis, precocidad $\mathrm{y}$ rendimiento del pimiento (Capsicum annun). Ciencia e Investigación Agraria 34 (3):195-204.

Di Rienzo J.A., F. Casanoves, M.G. Balzarini, L. Gonzalez, M. Tablada, y C.W. Robledo. 2013. InfoStat versión 2013. Disponible en http:// www.infostat.com.ar (Consulta 5 de marzo de 2015).

Domínguez, J., C. Lazcano, y M. Brandón. 2010. Influencia del vermicompost en el crecimiento de plantas. Aportes para la elaboración de un concepto objetivo. Acta Zoológica Mexicana 26(2):359-371.

Fermino, M.H. 2003. Métodos de análise para caracterização física de substratos para plantas. Tesis de Doctorado. Universidade Federal do Rio Grande, Rio Grande, Brasil.

López A.F., E. Chamorro, L. Vergara, y E. Utges. 1999. Estudio del lombricompuesto obtenido por biotransformación de lodos de curtiembre al tanino. Asades. Avances en Energías Renovables y Medio Ambiente 3:2831.

Melgar-Ramírez, R., y M.I. Pascual-Alex. 2010. Characterization and use of a vegetable waste vermicompost as an alternative component in substrates for horticultural seedbeds. Spanish Journal of Agricultural Research 8(4):1174-1182.

Noguera, P., M. Abad, R. Puchades, A. Maqueira, y V. Noguera. 2003. Influence of particle size on physical and chemical properties of coconut coir dust as container medium. Communications in Soil Science and Plant Analysis 34:593-605.

Paul, L.C., y J.D. Metzger. 2005. Impact of vermicompost on vegetable transplant quality. HortScience 40(7):2020-2023.

Restrepo, A.P., E. Medina, A. Pérez-Espinosa, E. Agulló, M.A. Bustamante, C. Mininni, et al. 2013. Substitution of peat in horticultural seedlings: suitability of digestate-derived compost from cattle manure and maize silage codigestion. Communications in Soil Science and Plant Analysis 44:668-677.

Tatlari, M., V. Abdossi, y Z.O. Ardebili. 2013. The effects of different levels of vermicompost on growth and development of Dracaena marginata. International Research Journal of Applied and Basic Sciences 4(4):784-786. 
Tringosvska, I., y T. Dintcheva. 2012. Vermicompost as substrate amendment for tomato transplant production. Sustainable Agriculture Research 1(2):115-122.

Valenzuela, O.R., V.H. Lallana, y A. Guerrero. 1998. Caracterización física y química de lombricompuestos originados a partir de residuos de conejeras, estiércol vacuno y residuos domiciliarios. Revista Científica Agropecuaria 2(1):45-48. 